\title{
25 Research Soure \\ Identification of microRNAs associated with heart failure with reduced ejection fraction
}

Xinli Li ( $\nabla$ xinli3267@njmu.edu.cn )

the First Affiliated Hospital of Nanjing Medical University

Qingqing Zhu

the First Affiliated Hospital of Nanjing Medical University

Mengli Chen

the First Affiliated Hospital of Nanjing Medical University

lokfai Cheang

the First Affiliated Hospital of Nanjing Medical University

Mengsha Shi

the First Affiliated Hospital of Nanjing Medical University

Ting Zhang

the First Affiliated Hospital of Nanjing Medical University

Rongrong Gao

the First Affiliated Hospital of Nanjing Medical University

Wenming Yao

the First Affiliated Hospital of Nanjing Medical University

\section{Research Article}

Keywords: Heart failure, Heart failure with reduced ejection fraction, miRNA

Posted Date: February 24th, 2022

DOI: https://doi.org/10.21203/rs.3.rs-1312127/v1

License: (c) (i) This work is licensed under a Creative Commons Attribution 4.0 International License.

Read Full License 


\section{Abstract}

Heart failure with reduced ejection fraction (HFrEF) is a complex disease with high mortality. The identification of new biomarkers and regulatory mechanisms for heart failure (HF) remains an important task. The crucial role of miRNAs in regulating the pathophysiological processes of HF has continuously been explored. Notably, the miRNA profiles from cardiac tissue and blood of patients with HFrEF were collected from the Gene Expression Omnibus (GEO) database in this study. After screening the data, two miRNAs (hsa-miR-216a and hsa-miR-217) with the highest cross-validation expression in tissues and blood and one miRNA (hsa-miR-208a) with the highest cross-validation expression in blood were identified. The miRNA-mRNA target gene interaction network and the enrichment analysis of the gene ontology (GO) and Kyoto Encyclopedia of Genomes (KEGG) pathways of differentially expressed miRNAs and target genes showed multiple signaling pathways with specific genes. The consistent expression of miR-217 in myocardial tissue and blood in HFrEF patients and mouse models demonstrates its reliability and repeatability in different etiologies, which will further deepen the understanding of miRNA as a potential biomarker and therapeutic target for heart failure.

\section{Introduction}

Heart failure (HF) is a complex clinical syndrome in the terminal stage of various cardiovascular diseases and is primarily characterized by ventricular filling, impaired ejection, or both, resulting in difficulty breathing or limited exertion. Heart failure with reduced ejection fraction (HFrEF) is determined by left ventricular ejection fraction (LVEF) $\leq 40 \%$ and is associated with high morbidity and mortality ${ }^{1,2}$. Novel and innovative biomarkers are urgently needed for the diagnosis and prognosis of HFrEF.

MicroRNAs (miRNAs) are noncoding RNAs approximately 22 nucleotides in length that inhibit the translation of multiple messenger RNAs (mRNAs) by binding to complementary 3' untranslated regions. The differential expression of miRNAs in various pathologies makes them potential diagnostic and prognostic biomarkers ${ }^{3}$ and has been shown to play a crucial role in HFrEF 4,5 . In addition to expression in tissues, miRNAs can be detected in blood and can be packaged in exosomes or microparticles or bind to proteins.

Difficult obtainment from valuable cardiac tissue limits the prediction of HFrEF by the detection of miRNA in tissue. Thus, most studies have only focused on circulating miRNAs in blood to reflect the full understanding of $\mathrm{HF}^{6,7}$. In addition, current studies have not simultaneously detected expression differences in the myocardium and blood. However, the expression characteristics of miRNAs in cardiac tissue, such as the coronary sinus (CS), were shown to be different compared with the blood liquid phase $^{8}$. Therefore, intersection analysis by a combination of existing data of the blood and myocardial tissue from different centers may be more helpful in understanding the biological link between miRNA expression and HF than traditional single-center blood screening among HFrEF patients. 
For this purpose, cardiac and blood samples containing HFrEF from three datasets in the Gene Expression Omnibus (GEO) database were downloaded in the present study. After identifying the intersectional differentially expressed miRNAs (DEmiRNAs) in datasets, the target genes of DEmiRNAs were predicted by multiple datasets. Gene ontology (GO) function and Kyoto Encyclopedia of Genomes (KEGG) pathway enrichment analyses were conducted on the basis of target genes and DEmiRNAs. Finally, protein-protein interaction (PPI) networks were established to investigate submodule analysis and hub genes in HFrEF. The expression differences of DEmiRNAs were further identified in the mouse HF model due to different etiologies to prove its reliability and repeatability and pave the way for the subsequent exploration of biological mechanisms.

\section{Results}

In the three expression profiles, we found the top two upregulated miRNAs (hsa-miR-216a and hsa-miR217) in both myocardial tissue and blood (shown in Table 1). Interestingly, the two belong to the same miRNA cluster on chromosome 2. In addition, hsa-miR-208a was the top upregulated miRNA, which was consistently upregulated in the blood in the two different datasets. Only hsa-miR-146b-3p showed a consistent expression trend of downregulated DEmiRNAs in both ventricular tissues and blood with the same expression profile. Therefore, no lists or further GO and KEGG analyses on downregulated DEmiRNAs were provided. All miRNAs were differentially expressed in the database and were repeated in different sample datasets [see Additional file 1], and the intersections of the different profiles are also shown [see Additional file 2].

\section{Target of DEmiRNAs}

A total of 713 genes were identified from the DEmiRNAs, among which the miRNA-targeted genes outlined the relationship shown in Figure 1. C5orf24 is the possible target gene of hsa-miR-216a-5p, hsamiR-217-3p, and hsa-miR-217-5p (as shown in Fig. 1).

\section{GO and KEGG Pathway Analyses of DEmiRNAs}

The enrichment data by GO and KEGG pathways showed that DEmiRNAs were associated with cellular components (CCs) involving "protein complexes" and "dendrites" and biological processes (BPs) involving "viral processes," "response to stress", "nucleobase-containing compound catabolic process", and "Toll-like receptor signaling pathway". In the "molecular function (MF)" category, "nucleic acid binding transcription factor activity", "protein binding transcription factor activity" and "protein binding, bridging" were the most frequent targets (Table 2). For KEGG pathway enrichment analysis, annotations were performed in the top 20 pathways according to the KEGG annotated gene number (Table 3 ). Most target genes were enriched in "TGF-beta signaling pathway", "morphine addiction", "signaling pathways regulating pluripotency of stem cells" and "thyroid hormone signaling pathway". 


\section{GO and KEGG Pathway Analyses of target mRNA}

The GO terms "cellular response to steroid hormone stimulus", "response to steroid hormone", "covalent chromatin modification" and "rhythmic process" in the BP category, "glutamatergic synapse, presynaptic membrane, synaptic membrane, ubiquitin ligase complex" in the CC category and "DNA-binding transcription activator activity", "RNA polymerase II-specific, ubiquitin-like protein transferase activity", and "protein phosphorylated amino acid binding" in the MF category accumulated the most frequent targets (Fig. 2A and 2B). Furthermore, KEGG analysis of candidate genes also showed that they were mainly enriched in the "FoxO signaling pathway", "TGF-beta signaling pathway" and "hormone signaling" pathways (Fig. 3A and 3B).

Specific enriched genes in "cellular response to steroid hormone stimulus", "histone modification", "response to steroid hormone", and "rhythmic process" were evaluated using enrichplot package in R and are presented [see Additional file 3].

\section{Identification of significant modules and genes}

The top 5 significant modules were selected from the PPI network, in which Molecular Complex Detection (MCODE) and cytoHubba were used to screen hub genes. Among them, UBE2N was identified by both MCODE and cytoHubba (Fig. 4), and 8 overlapping hub genes, including Fox03, KRAS, JAK2, MAPK, PTK2, PIK3R1, PTPN11, and SOS1, were also identified (Fig. 4 and Additional file 4).

\section{Validation of DEmiRNAs in different types of heart failure}

ANP and BNP were both overexpressed in the MI and TAC mouse models, which inferred the heart failure condition (Fig. 5A and Fig. 5B). The expression levels of miR-217-3p was increased in the TAC mouse model (Fig. 5C), and miR-217-5p expression was increased in the both MI model and TAC mouse model (Fig. 5D). However, no differential expression levels of other three miRNAs (miR-216-3p, miR-216-5p and miR-208a) were found between the control and the MI or TAC heart failure groups (Fig. 5E, Fig. 5F and Fig. $5 G)$.

\section{Discussion}

This study identified 5upregulatedDEmiRNAs (hsa-miR-216a-3p, hsa-miR-216a-5p, hsa-miR-217-3p, hsamiR-217-5p and hsa-miR-208a) in myocardial tissue and blood fromthemiRNA profiles of GSE135055, GSE53080, and GSE53437 and extracted consistentmiRNAswith multifold higher differential expression to distinguish HFrEF patients fromthecontrol population.miR-217 was also verifiedupregulatedinamouse model of heart failure caused by ischemia and hypertrophy. 
Hsa-miR-216a and hsa-miR-217 screened in this study have also been verified by previous studies screening circulating miRNAs as potential biomarkers for $\mathrm{HF}^{9}$. This study showed thatthecombination of miR-216a and miR-217 or more miRNAs could be considered a new diagnostic biomarker for HF.Previous studies have also shown increased expression of miR-217 in the hearts of CHF patients and mice with TAC surgery ${ }^{10}$. Our findings are consistent with these findings. Overexpression of miR-217 in vivo aggravated cardiac hypertrophy, fibrosis, and cardiac dysfunction caused by stress overload ${ }^{10,11}$. In addition, miR-217 wasupregulatedin ischemia-reperfusion model mice and in hypoxia/reoxygen-treated H9C2 cells ${ }^{12}$.Dual-specificity protein phosphatase 14 (DUSP14) has been shownto bea target, inactivatingtheNF-KB and MAPK pathways to prevent MI reperfusion injury ${ }^{12}$. Therefore, miR-217 could be a promising therapeutic target in $\mathrm{HF}$ cardiac hypertrophy mechanisms.

To date, miR-208has beenreportedto be amyocardium-specific miRNA ${ }^{7,13}$. Significant upregulation of miR-208a and related proteins was also presented in human hearts with type 2 diabetes ${ }^{14}$. However, its high expression was not observed in the myocardium of patients with HFrEF in the 3 profiles we included, but it was highly expressed in the blood samples, which was also verified intheplasma of HF patients in another study ${ }^{15}$.Together, no current evidence has shown miR-208a asaDEmiRNA in the myocardial tissue of MI and TAC mouse models,and this study also confirmedthis observation. However, miR-208a silencing can attenuate myocardial apoptosis and promote cardiac repair ${ }^{16}$, which needs further exploration.

Regarding the manifestation of the potential biological roles of 5 miRNAs in human heart failure, 9 key genes were enriched and analyzed, most of which were confirmed to be related to HF. JAK-STAT signaling is associated with cardiac hypertrophy and remodeling induced by pressure overload and ischemiareperfusion-induced cardiac dysfunction ${ }^{17}$. SOS1, PTPN11, and KRAS mutations may be associated with hypertrophic cardiomyopathy of RASopathy ${ }^{18}$. PIK3R1 is modulated bytheFoxO3a pathway, which is highly phosphorylated in dysfunctional hearts ${ }^{19}$. However, there are no relevant reports on the role of UBE2N (E2 ubiquitin-conjugating enzymes) in HFrEF and even in the heart. This study was the first toidentifyUBE2N not only as a hub gene but alsoasa key molecule in MCODE module 1 , which needs to be explored further.

Gene enrichment analysis revealed that several signaling pathwayswereinvolved in the regulation oftheTGF- $\beta$ signaling pathway, the FoxO signaling pathway, theToll-like receptor signaling pathway, stress response, and hormone signaling pathways,such as those for thyroid and steroid hormones, which arewidelyreported to correlate with $\mathrm{HF}^{20-23}$. Additionally, the signaling molecules in each pathway may becorrelated.FoxO3a has been shown to act as a negative regulator of TGF- $\beta 1$-induced cardiac extracellular matrix (ECM) transformation ${ }^{24}$, and TLR2 deficiency leads to excessive activation of the transcription factor Forkhead box protein 01 (Fox01), which affects age-related cardiac function in mice ${ }^{25}$.TLR4 activates the expression of severalproinflammatorycytokine genes in myocardial inflammation ${ }^{26}$. 
Our study has some limitations. First, the etiology of heart failure is not completely presented in this study,andonly the common etiology is constructed.Second, we did not choose to use mouse serum for verification.This isbecauseprevious studies have shown thatmiRNAsobserved in human patients with HF are mostly present at low levels and cannot be validated in well-established mouse models ${ }^{27}$.

This study may shed new light on the combination ofbiomarkersin HF by combining multiple expression profiles.Multi-miRNAs combined with or without BNP still significantlyimprovethe prediction model to distinguish heart failure with preserved ejection fraction (HFpEF) from HFrEF andimprovespecificity and accuracy ${ }^{28}$.Therefore, with increased sample size and reduced deviation of detection, this study ultimately improved the authenticity of the findings. In addition to preliminary verification at the animal level, the miRNA screened is expected to be used asapanel in the next step to distinguish HFrEFs in our center.

In conclusion, we identified valuable biomarkers in tissues and blood and established a miRNA-mRNA regulatory network. In addition, bioinformatics techniques were applied to explore the crucial molecules involved in the development of HFrEF. We found that miR-217 was consistently expressed in HFrEF through animal heart failure models, offering attractive potential as a biomarker and therapeutic target for heart failure.

\section{Methods}

\section{Source of expression profiles}

We downloaded the expression matrices of GSE135055, GSE53080, and GSE53437 from the GEO database (http://www.ncbi.nlm.nih.gov/geo/) after careful review of the patient stages. Samples of myocardial tissue were selected from $21 \mathrm{HFrEF}$ patients and 9 normal subjects in the GSE135055 dataset, $35 \mathrm{HFrEF}$ patients and 10 control samples in the GSE53080 dataset, and plasma samples of 68 HFrEFs and 21 controls were also included. We also selected plasma samples from $24 \mathrm{HFrEFs}$ and 14 controls from the GSE53437 dataset (GLP18067).

\section{Identification of DEmiRNAs}

DESeq2 was used to determine differentially expressed miRNAs in R 3.6.3. Differentially expressed miRNAs were identified by meeting the cutoff criteria of the adjusted $P$ value (adj. $P$ ) $<0.05$ and log2 FC $>2.0$. Additionally, miRNAs with the most significant fold change and present in both tissues and blood were chosen as DEmiRNAs in Table 1.

\section{Prediction of DEmiRNA target genes}


The TargetScan (http://www.targetscan.org/), miRDB (http://www.mirdb.org/) and mirDIP (http://ophid.utoronto.ca/mirDIP/) online databases were selected, and genes identified in all three datasets were regarded as DEmiRNA targets. However, the miR-217-3p-related data were only present in two databases (miRDB and mirDIP). Therefore, the intersection of the two databases was selected as the targeted gene. DEmiRNAs and target genes were selected to construct a miRNA-mRNA regulatory network using Cytoscape software 3.8 and Metascape (http://metascape.org/).

\section{Functional GO and KEGG enrichment analyses of miRNAs}

We used DIANA TOOLS mirPath (http://diana.imis.athena-innovation.gr/DianaTools/index.php) to perform the KEGG pathway enrichment analysis and the $\mathrm{GO}$ enrichment consisting of biological processes (BP), molecular functions (MF) and cellular components (CC). According to hypergeometric distribution theory, the enrichment threshold of $G O$ and KEGG items was set as $P<0.05$. In addition, we used R 3.6.3 for KEGG and GO enrichment analyses of the target gene set. $\mathrm{P}<0.05$ was considered statistically significant.

\section{PPI network and modules analysis}

Protein interactions among the target genes of DEmiRNAs were analyzed using the STRING online database. Ultimately, a total of 258 nodes and 872 edges were identified from the PPI network program. Subsequently, the network was visualized in Cytoscape, and the top 5 significant gene modules were selected from the PPI network using Molecular Complex Detection (MCODE). In addition, the top 10 genes were selected and ranked by closeness and degree using cytoHubba.

\section{Animal models}

\section{Myocardial infarction and transverse aortic constriction surgery-induced HF models}

All experimental procedures were conducted in accordance with relevant guidelines and the National Institutes of Health Guide for the Care and Use of Laboratory Animals. The experiments complied with the ARRIVE guidelines (https://arriveguidelines.org). The procedures for the care and use of animals were approved by the Ethics Committee of The Nanjing Medical University (no. IACUC-1903016). Male C57BL/ 6 mice (aged 7-8 weeks old, weighing 18-20 g) were used to establish a myocardial infarction (MI) model and a transverse aortic constriction (TAC) model. MI was generated by ligating the left anterior descending coronary artery (LAD) using a 7-0 nylon suture, while sham was created by the same process but without LAD ligation. During TAC, after separating the aortic arch between the innominate artery and the left common carotid artery, a 7-0 nylon suture was tightened three 
times. The same intervention was performed in the sham mice except for aortic contraction. After 4 weeks, the mice were sacrificed, and their hearts were removed. ANP and BNP tests confirmed the presence of heart failure in mice.

\section{Quantitative real-time polymerase chain reaction (qRT-PCR)}

Total RNA extracted from ventricular tissues was used for miRNA Quantitative Real-time Polymerase Chain Reactions (qRT-PCRs) following reverse transcription using a Bio-Rad iScript ${ }^{T M}$ cDNA Synthesis Kit (Bio-Rad, Hercules, CA, USA) to obtain cDNAs. The expression levels of ANP and BNP were analyzed using a Bio-Rad SYBR qPCR kit (Bio-Rad, Hercules, CA, USA) on an ABI-7900 Real-Time PCR Detection System (7900HT, Applied Biosystems, CA, USA). For miRNA, the Bulge-Loop ${ }^{T M}$ miRNA qPCR Primer Set (RiboBio) was used to determine the expression levels of miRNAs with Takara SYBR Premix Ex Taq ${ }^{\text {TM }}$ (Tli RNaseH Plus) on an ABI-7900 Real-Time PCR Detection System (Applied Biosystems). The relative miRNA expression was normalized to $U 6$ levels and calculated through the $2-{ }^{\Delta} \mathrm{Ct}$ method, while $18 \mathrm{~S}$ RNA was used as an internal control for ANP and BNP expression.

Mouse-ANP: F: AGGCAGTCGATTCTGCTT, R: CGTGATAGATGAAGGCAGGAAG;

Mouse-BNP: F: TAGCCAGTCTCCAGAGCAATTC, R: TTGGTCCTTCAAGAGCTGTCTC;

Mouse-18S: F: TCAAGAACGAAAGTCGGAGG, R: GGACATCTAAGGGCATCAC.

\section{Statistical analysis}

All experimental data were analyzed using SPSS software (version 23.0) and are presented as the mean \pm SD (using GraphPad Prism 8.0). An independent-samples t test was used to compare two groups. Significance of $P<0.05$ is indicated by *, and $P<0.01$ by **.

\section{Declarations}

\section{Author contributions}

Q.Z. and M.C. conceived the work and revised the manuscript. I.C. and R.G. performed the bioinformatics analysis. W.Y. and X.L. designed the experiments and drafted the manuscript. M.S. and T.Z. finished the experiments. All authors have read and approved the final manuscript.

\section{Funding}

This work was supported by Key Program of National Natural Science Foundation of China (81730106 to XL Li), General Program of National Natural Science Foundation of China (81970339 to XL Li), The 
National High Technology Research and Development Program of China (2017YFC1700505 to XL Li), and Natural Science Foundation project of Jiangsu (BK20191072 to RR Gao).

\section{Data availability}

The datasets generated and/or analyzed during the current study are available in the Gene Expression Omnibus (GEO) repository, GSE135055, GSE53080, and GSE53437. All data supporting the findings of the study are included in this published article (and its Supplementary Information files).

\section{Additional information}

\section{Competing interests}

The authors declare that they have no competing interests.

\section{References}

1. Shah K.S., et al. Heart Failure With Preserved, Borderline, and Reduced Ejection Fraction: 5-Year Outcomes. J Am Coll Cardiol. 70, 2476-2486 (2017).

2. Murphy S.P., Ibrahim N.E. \& Januzzi J.L., Jr. Heart Failure With Reduced Ejection Fraction: A Review. Jama. 324, 488-504 (2020).

3. Vogel B., et al. Multivariate miRNA signatures as biomarkers for non-ischaemic systolic heart failure. Eur Heart J. 34, 2812-2822 (2013).

4. Chen Y.T., Wong L.L., Liew O.W. \& Richards A.M. Heart Failure with Reduced Ejection Fraction (HFrEF) and Preserved Ejection Fraction (HFpEF): The Diagnostic Value of Circulating MicroRNAs. Cells. 8 (2019).

5. Vegter E.L., van der Meer P., de Windt L.J., Pinto Y.M. \& Voors A.A. MicroRNAs in heart failure: from biomarker to target for therapy. Eur J Heart Fail. 18, 457-468 (2016).

6. Zhirov I.V., et al. [Full - transcriptome analysis of miRNA expression in mononuclear cells in patients with acute decompensation of chronic heart failure of various etiologies]. Ter Arkh 91, 62-67 (2019).

7. Li D.M., et al. Diagnostic value of circulating microRNA-208a in differentiation of preserved from reduced ejection fraction heart failure. Heart Lung. 50, 71-74 (2021).

8. Ben-Zvi I., et al. Cardiac-peripheral transvenous gradients of microRNA expression in systolic heart failure patients. ESC Heart Fail. 7, 835-843 (2020).

9. Ding H., et al. Combined detection of miR-21-5p, miR-30a-3p, miR-30a-5p, miR-155-5p, miR-216a and miR-217 for screening of early heart failure diseases. Biosci Rep. 40 (2020). 
10. Nie X., et al. miR-217 Promotes Cardiac Hypertrophy and Dysfunction by Targeting PTEN. Mol Ther Nucleic Acids. 12, 254-266 (2018).

11. Thienpont B., et al. The H3K9 dimethyltransferases EHMT1/2 protect against pathological cardiac hypertrophy. J Clin Invest. 127, 335-348 (2017).

12. Li Y., Fei L., Wang J. \& Niu Q. Inhibition of miR-217 Protects Against Myocardial IschemiaReperfusion Injury Through Inactivating NF-KB and MAPK Pathways. Cardiovasc Eng Technol. 11, 219-227 (2020).

13. Zhao X., Wang Y. \& Sun X. The functions of microRNA-208 in the heart. Diabetes Res Clin Pract. 160, 108004 (2020).

14. Rawal S., Nagesh P.T., Coffey S., Van Hout I., Galvin I.F., Bunton R.W., Davis P., Williams M.J.A. \& Katare R. Early dysregulation of cardiac-specific microRNA-208a is linked to maladaptive cardiac remodelling in diabetic myocardium. Cardiovasc Diabetol. 18, 13 (2019).

15. Mohammadi A., et al. Evaluation of Oxidative Stress, Apoptosis, and Expression of MicroRNA-208a and MicroRNA-1 in Cardiovascular Patients. Rep Biochem Mol Biol. 10, 183-196 (2021).

16. Tony H., et al. MicroRNA-208a Dysregulates Apoptosis Genes Expression and Promotes Cardiomyocyte Apoptosis during Ischemia and Its Silencing Improves Cardiac Function after Myocardial Infarction. Mediators Inflamm. 2015, 479123 (2015).

17. Booz G.W., Day J.N. \& Baker K.M. Interplay between the cardiac renin angiotensin system and JAKSTAT signaling: role in cardiac hypertrophy, ischemia/reperfusion dysfunction, and heart failure. $J$ Mol Cell Cardiol. 34, 1443-1453 (2002).

18. Chen $\mathrm{H}$., et al. Clinical and mutation profile of pediatric patients with RASopathy-associated hypertrophic cardiomyopathy: results from a Chinese cohort. Orphanet J Rare Dis. 14, 29 (2019).

19. Kumarswamy R., et al. SERCA2a gene therapy restores microRNA-1 expression in heart failure via an Akt/FoxO3A-dependent pathway. Eur Heart J. 33, 1067-1075 (2012).

20. Yue Y., Meng K., Pu Y. \& Zhang X. Transforming growth factor beta (TGF- $\beta$ ) mediates cardiac fibrosis and induces diabetic cardiomyopathy. Diabetes Res Clin Pract. 133, 124-130 (2017).

21. Yao Y., et al. ADAMTS16 activates latent TGF- $\beta$, accentuating fibrosis and dysfunction of the pressure-overloaded heart. Cardiovasc Res. 116, 956-969 (2020).

22. Auguste G., et al. Suppression of Activated FOXO Transcription Factors in the Heart Prolongs Survival in a Mouse Model of Laminopathies. Circ Res. 122, 678-692 (2018).

23. Okada K., et al. Wnt/ $\beta$-Catenin Signaling Contributes to Skeletal Myopathy in Heart Failure via Direct Interaction With Forkhead Box O. Circ Heart Fail. 8, 799-808 (2015)

24. Vivar R., et al. Role of FoxO3a as a negative regulator of the cardiac myofibroblast conversion induced by TGF- $\beta 1$. Biochim Biophys Acta Mol Cell Res. 1867, 118695 (2020).

25. Spurthi K.M., et al. Toll-like receptor 2 deficiency hyperactivates the FoxO1 transcription factor and induces aging-associated cardiac dysfunction in mice. J Biol Chem. 293, 13073-13089 (2018). 
26. Yang Y., et al. The emerging role of Toll-like receptor 4 in myocardial inflammation. Cell Death Dis. 7, e2234 (2016).

27. Vegter E.L., et al. Rodent heart failure models do not reflect the human circulating microRNA signature in heart failure. PLoS One. 12, e0177242 (2017).

28. Wong L.L., et al. Circulating microRNAs in heart failure with reduced and preserved left ventricular ejection fraction. Eur J Heart Fail. 17, 393-404 (2015).

\section{Tables}

\section{Table 1}

The most differentially up-regulated microRNAs in patients with HFrEF. hsa-miR-216a and hsa-miR-217 belong to a microRNA cluster. $P$ is adjusted by FDR. HFrEF: heart failure with reduced left ventricular ejection fraction. These MicroRNAs are all up-regulated. FDR: false discovery rate.

\begin{tabular}{|llllll|}
\hline MicroRNA & log2FoldChange & Padj & $\begin{array}{l}\text { GSE } \\
\text { number }\end{array}$ & Sample type & Methods \\
\hline $\begin{array}{l}\text { hsa-miR- } \\
216 a-3 p\end{array}$ & 3.033864 & 0.006388 & GSE53080 & $\begin{array}{l}\text { left-ventricular } \\
\text { myocardium }\end{array}$ & $\begin{array}{l}\text { RNA- } \\
\text { sequencing }\end{array}$ \\
$\begin{array}{l}\text { hsa-miR- } \\
216 a-3 p\end{array}$ & 2.404703 & 0.014913 & GSE135055 & $\begin{array}{l}\text { left ventricular } \\
\text { heart tissue }\end{array}$ & $\begin{array}{l}\text { RNA- } \\
\text { sequencing }\end{array}$ \\
\hline $\begin{array}{l}\text { hsa-miR- } \\
216 a-5 p\end{array}$ & 4.153001 & $3.86 E-05$ & GSE135055 & $\begin{array}{l}\text { left ventricular } \\
\text { heart tissue }\end{array}$ & $\begin{array}{l}\text { RNA- } \\
\text { sequencing }\end{array}$ \\
\hline $\begin{array}{l}\text { hsa-miR- } \\
216 a-5 p\end{array}$ & 5.732168 & $6.79 E-12$ & GSE53080 & $\begin{array}{l}\text { left-ventricular } \\
\text { myocardium }\end{array}$ & $\begin{array}{l}\text { RNA- } \\
\text { sequencing }\end{array}$ \\
$\begin{array}{l}\text { hsa-miR- } \\
216 a-5 p\end{array}$ & 5.792320719 & $4.91 E-10$ & GSE53080 & Plasma & $\begin{array}{l}\text { RNA- } \\
\text { sequencing }\end{array}$ \\
\hline $\begin{array}{l}\text { hsa-miR-217- } \\
\text { 3p }\end{array}$ & 3.265644 & 0.00886 & GSE53080 & $\begin{array}{l}\text { left-ventricular } \\
\text { myocardium }\end{array}$ & $\begin{array}{l}\text { RNA- } \\
\text { sequencing }\end{array}$ \\
\hline $\begin{array}{l}\text { hsa-miR-217- } \\
5 p\end{array}$ & 3.595953 & $8.82 E-08$ & GSE135055 & $\begin{array}{l}\text { left ventricular } \\
\text { heart tissue }\end{array}$ & $\begin{array}{l}\text { RNA- } \\
\text { sequencing }\end{array}$ \\
\hline $\begin{array}{l}\text { hsa-miR-217- } \\
5 p\end{array}$ & 3.560764 & $1.79 E-07$ & GSE53080 & $\begin{array}{l}\text { left-ventricular } \\
\text { myocardium }\end{array}$ & $\begin{array}{l}\text { RNA- } \\
\text { sequencing }\end{array}$ \\
\hline $\begin{array}{l}\text { hsa-miR-217- } \\
5 p\end{array}$ & 3.520374167 & $9.81 E-06$ & GSE53080 & Plasma & $\begin{array}{l}\text { RNA- } \\
\text { sequencing }\end{array}$ \\
\hline $\begin{array}{l}\text { hsa-miR- } \\
208 a\end{array}$ & 5.496002228 & $1.25 E-18$ & GSE53080 & peripheral blood & $\begin{array}{l}\text { RNA- } \\
\text { sequencing }\end{array}$ \\
\hline $\begin{array}{l}\text { hsa-miR- } \\
208 a\end{array}$ & 2.399704182 & 0.025832596 & GSE53437 & plasma & $\begin{array}{l}\text { microRNA } \\
\text { Array }\end{array}$ \\
\hline
\end{tabular}


Table 2

Enriched GO terms for DEmiRNA in HFrEF by MirPath v.3. GO: Gene Ontology.

\begin{tabular}{|lll|}
\hline GO Category & log10(p-value) & genes \\
\hline Cellular component & & \\
\hline Protein complex & 3.102526 & 190 \\
\hline Dendrite & 0.312393 & 33 \\
\hline Molecular function & & \\
\hline Nucleic acid binding transcription factor activity & 14.04078 & 137 \\
\hline Protein binding transcription factor activity & 3.654436 & 52 \\
\hline Protein binding, bridging & 1.89647 & 18 \\
\hline Enzyme binding & 1.829344 & 73 \\
\hline Cytoskeletal protein binding & 0.867238 & 25 \\
\hline Biological processes & & 29 \\
\hline Viral process & 1.619838 & 29 \\
\hline Response to stress & 1.469473 & 122 \\
\hline Nucleobase-containing compound catabolic process & 1.348542 & 29 \\
\hline Catabolic process & 1.162052 & 55 \\
\hline Toll-like receptor 10 signaling pathway & 0.836023 & 7 \\
\hline Toll-like receptor TLR1:TLR2 signaling pathway & 0.725055 & 7 \\
\hline Toll-like receptor TLR6:TLR2 signaling pathway & 0.725055 & 7 \\
\hline Signal transduction & 0.658639 & 275 \\
\hline Regulation of focal adhesion assembly & 0.560604 & 4 \\
\hline Camp catabolic process & 0.354128 & 6 \\
\hline
\end{tabular}

\section{Table 3}

Top 20 Enriched KEGG pathways for DEmiRNA in HFrEF by MirPath v.3. KEGG pathway analysis of the top predicted differentially expressed miRNAs. KEGG: Kyoto Encyclopedia of Genes and Genomes; FDR: false discovery rate. 


\begin{tabular}{|lll|}
\hline KEGG Pathway & -log10(p-value) & genes \\
\hline TGF-beta signaling pathway & 5.76688784 & 14 \\
\hline Morphine addiction & 5.51688891 & 20 \\
\hline Signaling pathways regulating pluripotency of stem cells & 3.08824944 & 25 \\
\hline Thyroid hormone signaling pathway & 2.55270922 & 15 \\
\hline Proteoglycans in cancer & 2.38803954 & 29 \\
\hline GABAergic synapse & 1.93823963 & 4 \\
\hline Thyroid hormone synthesis & 1.81435757 & 2 \\
\hline Nicotine addiction & 1.74250958 & 7 \\
\hline FoxO signaling pathway & 1.50592531 & 10 \\
\hline Fatty acid degradation & 1.47926985 & 2 \\
\hline MicroRNAs in cancer & 1.337017243 & 13 \\
\hline Mucin type O-Glycan biosynthesis & 1.298318233 & 3 \\
\hline Glycosaminoglycan biosynthesis - heparan sulfate / heparin & 1.148768994 & 2 \\
\hline Retrograde endocannabinoid signaling & 1.070252565 & 14 \\
\hline ErbB signaling pathway & 1.008057575 & 14 \\
\hline Glioma & 1.00610234 & 5 \\
\hline Dorso-ventral axis formation & 0.995637349 & 7 \\
\hline Choline metabolism in cancer & 0.94910427 & 14 \\
\hline Rap1 signaling pathway & 0.939230413 & 25 \\
\hline Glycosphingolipid biosynthesis - lacto and neolacto series & 0.611397091 & 1 \\
\hline & & \\
\hline
\end{tabular}

\section{Figures}



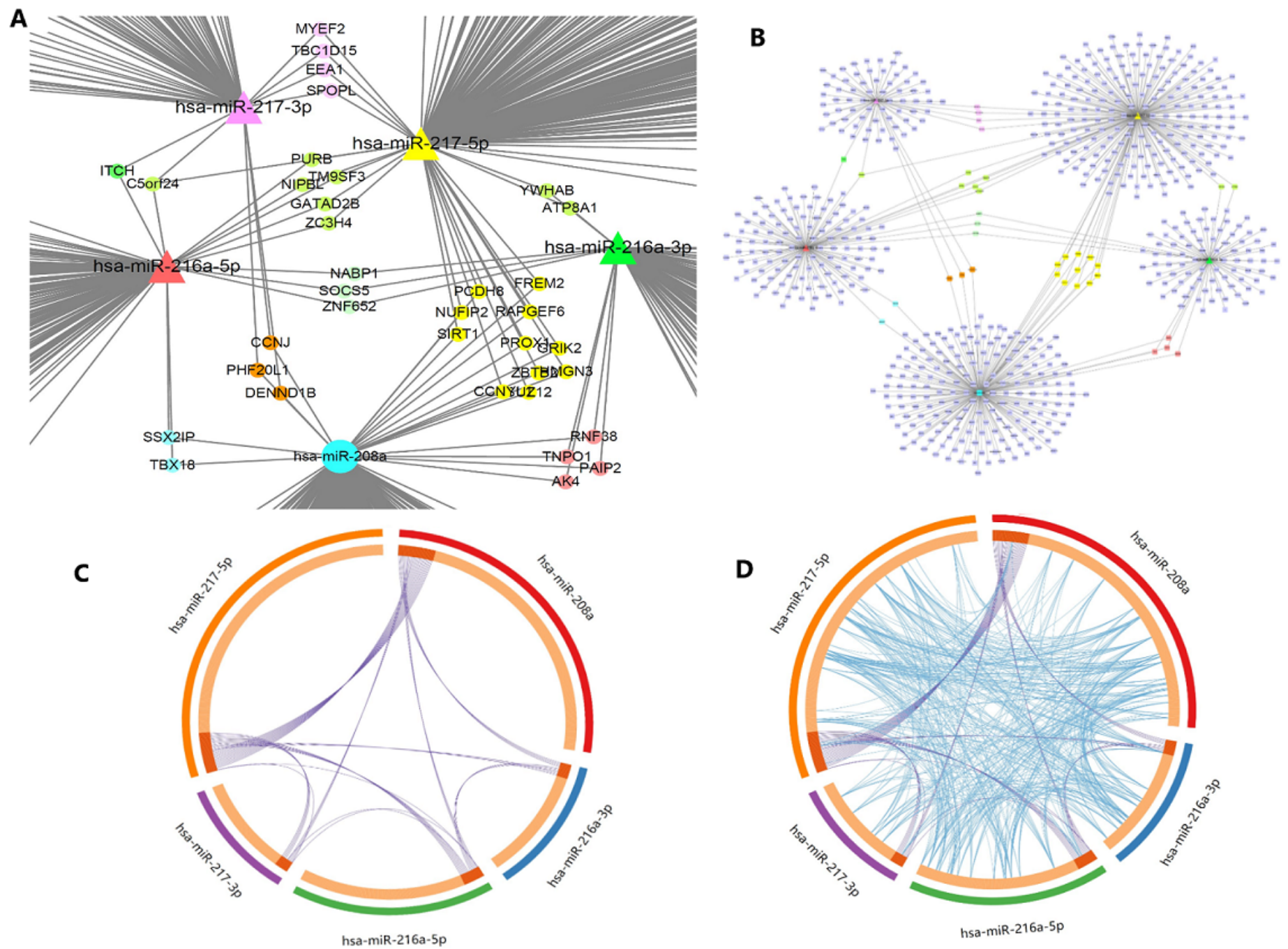

\section{Figure 1}

DEmiRNAs-mRNA regulatory network. (A) DEmiRNAs and miRNA-targeted genes identified by TargetScan, miRDB and mirDIP are shown by Cytoscape. (B) Overall view of DEmiRNAs and miRNAtargeted genes. (C) The synteny relationships of DEmiRNAs are shown by Metascape only at the gene level, with purple curves linking the same genes. (D) The synteny relationships of DEmiRNAs are shown, including GO term levels, where the blue curves link genes that belong to the same GO term-enriched genes. The inner circle represents the list of genes, and the outer circle represents the different DEmiRNAs. The overlapping genes are colored dark orange, while genes that do not overlap are shown in light orange. 
A

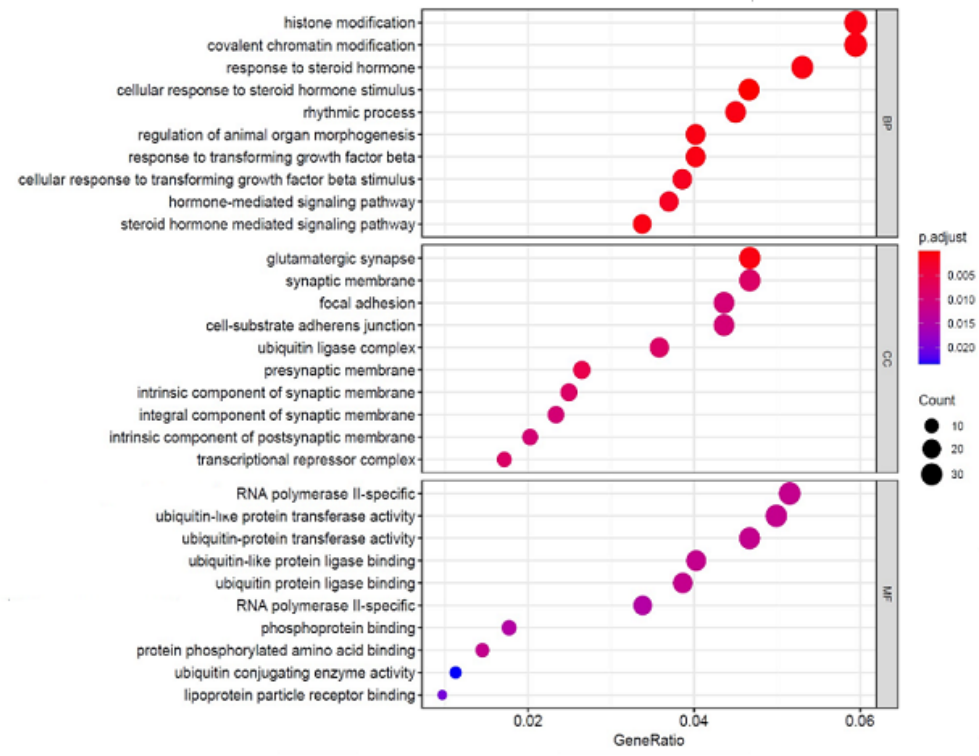

B

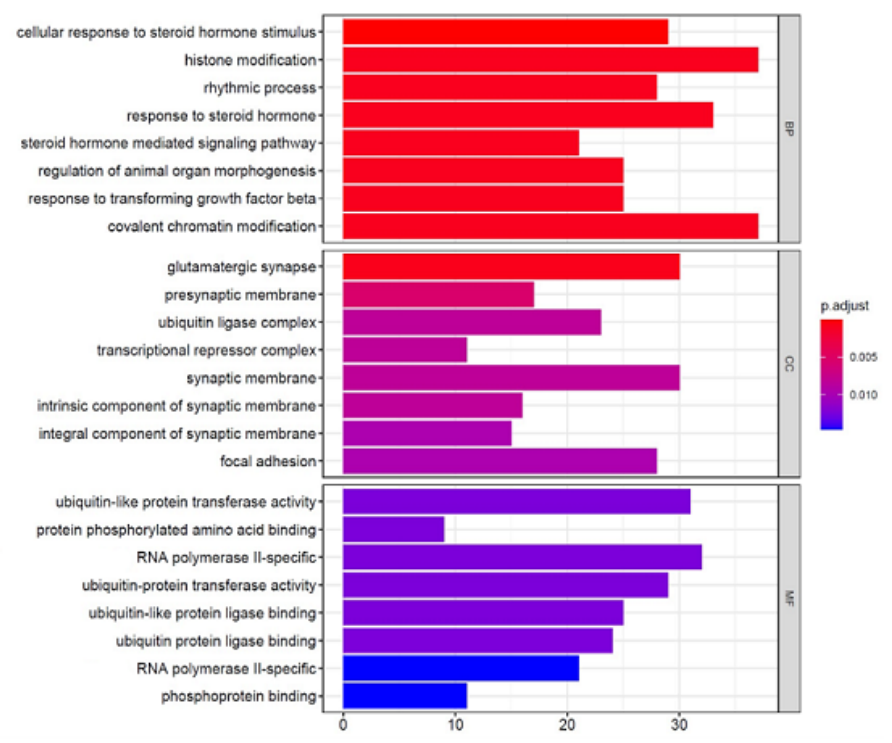

GO

Figure 2

GO analyses of the 713 targeted genes. (A) Dot and (B) bar plots of GO analyses. (A) The dot plot shows the GO enrichment analysis: the horizontal axis represents the gene ratio (the proportion of the number of screened gene sets to background genes), and the vertical axis is the detailed information of the GO terms. (B) GO analysis is shown in a bar chart, and the horizontal axis indicates the specific number of genes involved. 
A

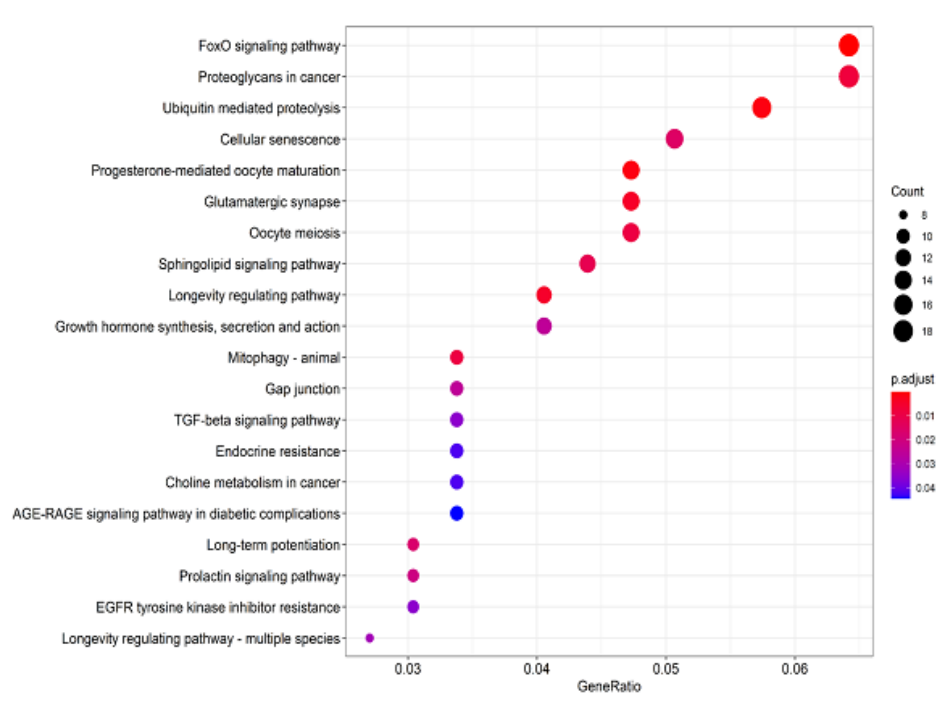

B

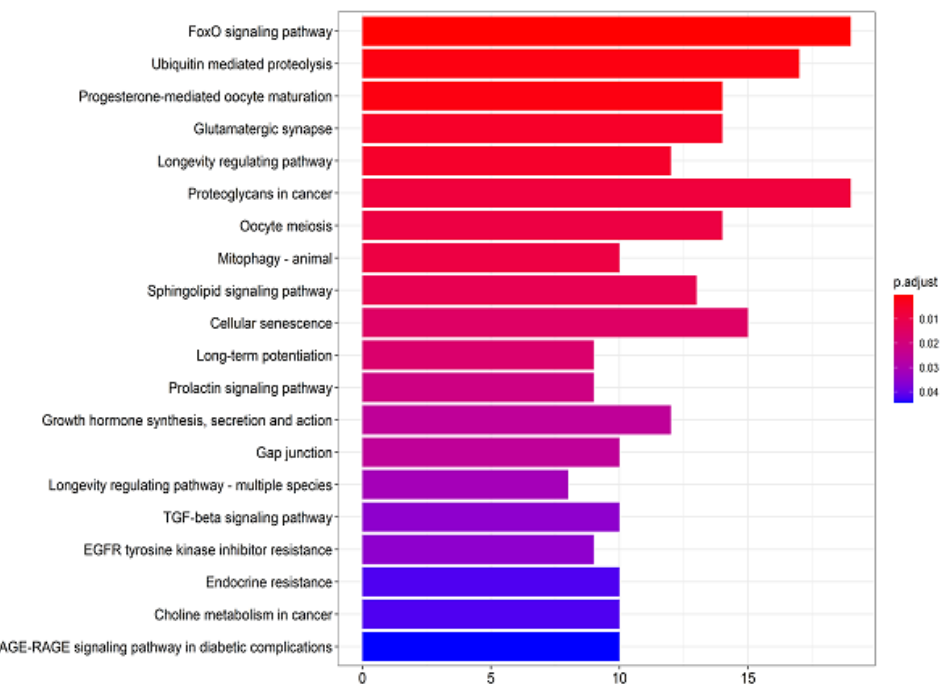

\section{KEGG}

\section{Figure 3}

KEGG analyses of the 713 targeted genes. (A) Dot and (B) bar plots of KEGG analyses. (A) The dot plot shows the KEGG enrichment analysis. The horizontal axis represents the gene ratio (the proportion of the number of screened gene sets to background genes), and the vertical axis is detailed information on the KEGG pathways. (B) The horizontal axis indicates the specific number of genes involved. 
A

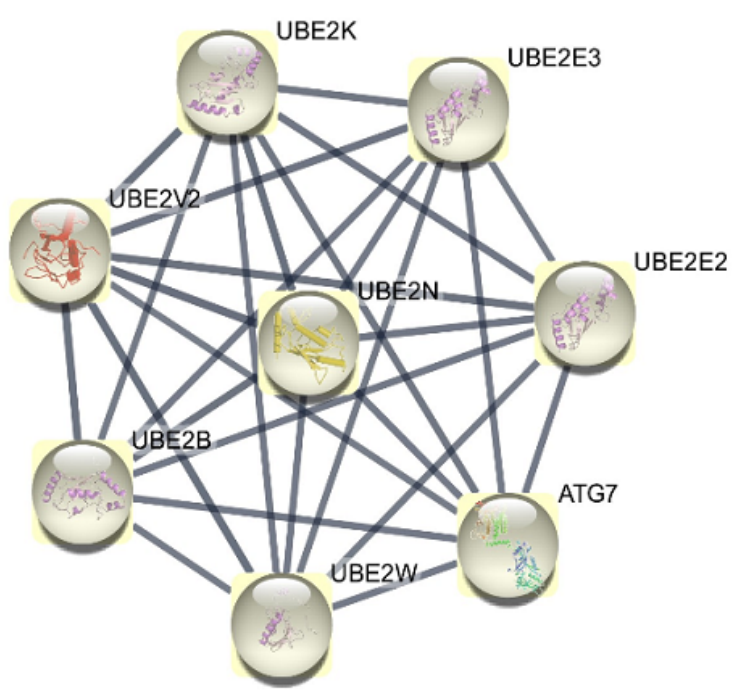

B

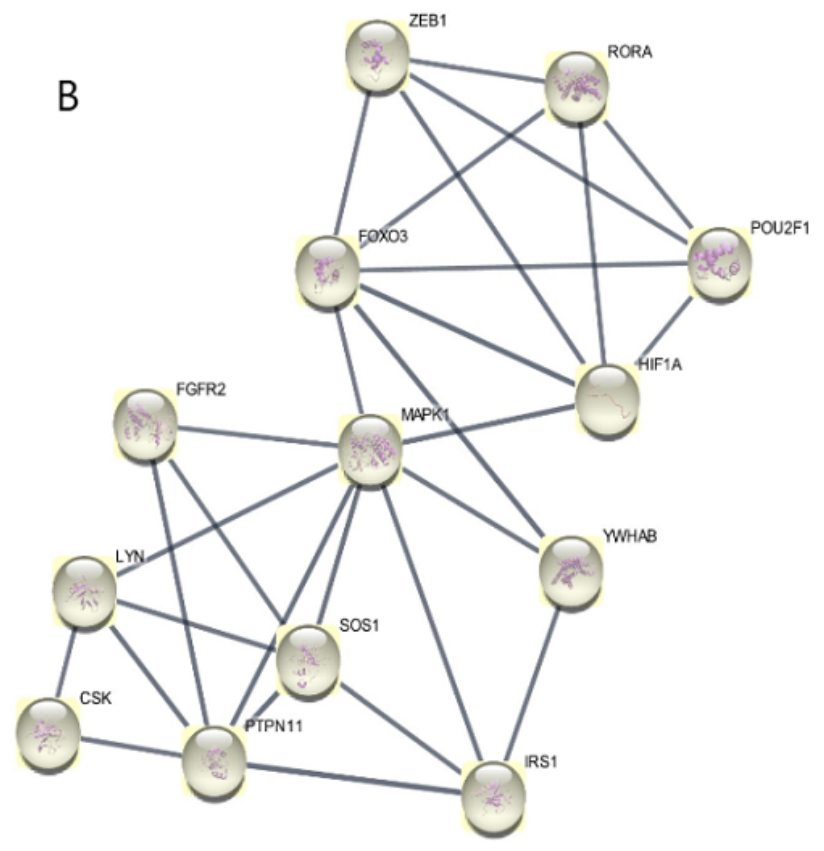

E

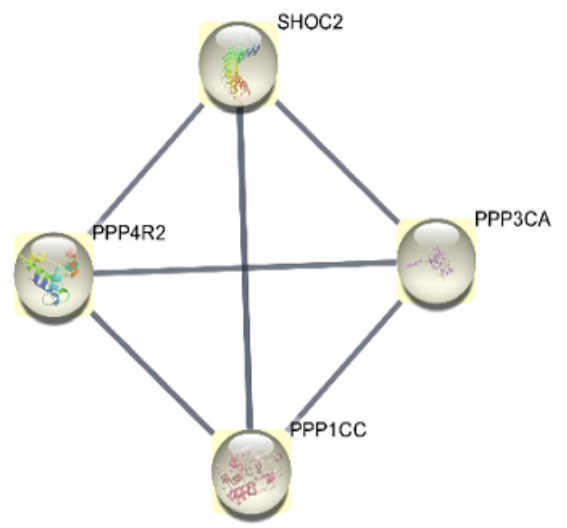

Figure 4

The top 5 significant modules identified from the PPI network by using MCODE. (A) Module A, (B) module $B,(C)$ module $C,(D)$ module $D$, and (E) module $E$. 

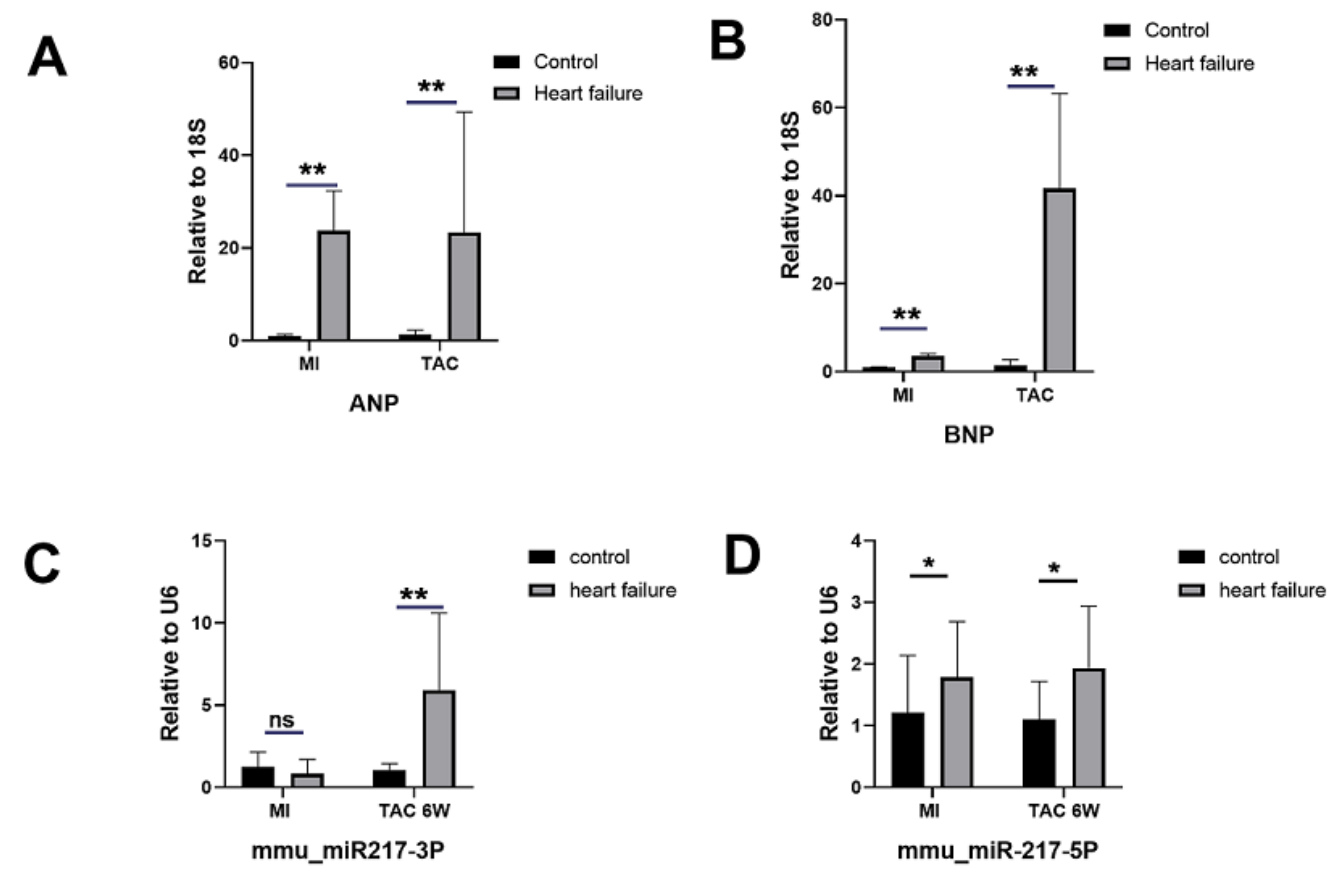

E

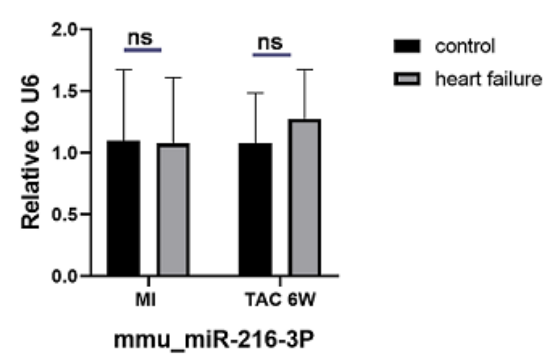

$\mathbf{F}$

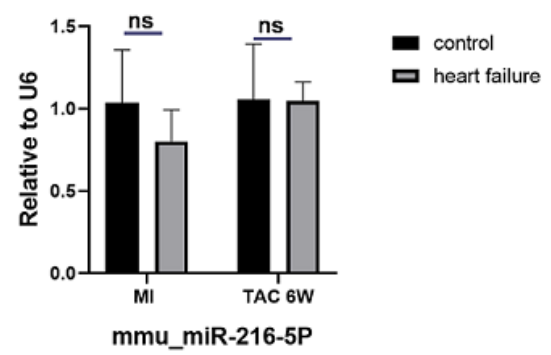

G

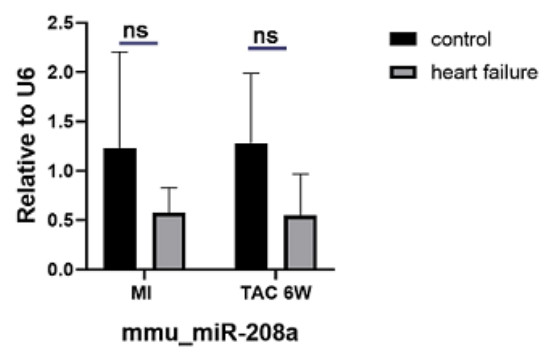

Figure 5

DEmiRNAs and ANP and BNP expression in MI and TAC mice model. (A, B) ANP and BNP expression in $\mathrm{MI}(\mathrm{n}=6)$ and TAC $(\mathrm{n}=6)$ model; $(\mathrm{C}, \mathrm{D}, \mathrm{E}, \mathrm{F}, \mathrm{G})$ mmu-miR-217-3p, mmu-miR-217-5p, mmu-miR-216a-3p, mmu-miR-216a-5p and mmu-miR-208a expression in MI and TAC model; Results are presented as fold changes (mean $\pm S D$ ) relative to sham group $(n=6)$. Delta CTs were obtained after normalization on U6 level. $\mathrm{SD}=$ standard deviation; $\mathrm{CT}=$ threshold cycle number. ${ }^{*} \mathrm{P}, 0.05 ;{ }^{*} \mathrm{P}, 0.01 ; \mathrm{ns}=$ not significant.

\section{Supplementary Files}

This is a list of supplementary files associated with this preprint. Click to download.

- Sulpplymentarytable.docx

- SupplementaryInformation.docx 
- Supplymentaryfig1.png

- Supplymentaryfig2.tiff

- Supplymentaryfig3.tiff 